\title{
Comparison of the maximum likelihood and Bayes estimators for symmetric bivariate exponential distribution under different loss functions
}

\author{
Assia Chadli ${ }^{\dagger}$, Hamida Talhi ${ }^{\dagger}$ and Hocine Fellag ${ }^{\ddagger, *}$ \\ ${ }^{\dagger}$ Badji-Mokhtar University Department of Mathematics B.P.12, Annaba 23000. Algeria \\ ${ }^{\ddagger}$ Laboratory of Pure and Applied Mathematics Mouloud Mammeri University of Tizi-Ouzou. Tizi- \\ Ouzou. 15000. Algeria
}

Received: June 6, 2013; Accepted: October 7, 2013

Copyright (C) 2013, Afrika Statistika. All rights reserved

\begin{abstract}
Consider a system with lifetime governed by a bivariate exponential model $(X, Y)$. Using the maximum likelihood method and the Bayesian approach, we estimate the parameters and the mean time between failure (MTBF) of this model.To compare the estimators, an exhaustive Monte Carlo study is performed using the Pitman closeness criterion and the relative efficiency.

Résumé. Considérons un système dont la durée de vie est régie par un modèle exponentiel bivarié $(X, Y)$. En utilisant la méthode du maximum de vraisemblance et l'approche Bayesienne, nous estimons les paramètres, le temps moyen de bon fonctionnement (MTBF) et la fonction de fiabilité de ce modèle. Une étude de Monte Carlo est menée pour comparer les estimateurs moyennant les critères de proximité de Pitman et d'efficacité relative.
\end{abstract}

Key words: Bayesian Analysis; Bivariate Exponential Model; Loss function; Maximum Likelihood Estimation; Reliability.

AMS 2010 Mathematics Subject Classification : 62M20; 62 N05.

\section{Introduction}

The exponential distribution denoted by $E x$ plays an important role in survival problems since it has a constant failure rate and it has no memory. Many bivariate models are derived

*Corresponding author Hocine Fellag: hfellag@yahoo.com Assia Chadli : assiachadli428@hotmail.com

Hamida Talhi : talhihamida@yahoo.fr 
A. Chadli, H. Talhi and H. Fellag, Afrika Statistika, Vol. 8, 2013, pages 499-514. Comparison of the maximum likelihood and Bayes estimators for symmetric bivariate exponential distribution under different loss functions.

from the exponential model. Block and Basu (1974) considered the maximum likelihood estimator (MLE) of the Absolutely Continuous Bivariate Exponential (ACBVE) and Weier (1981) proposed a Bayesian estimator of the reliability function using a noninformative and conjugate priors. Klein and Basu (1985) considered the estimation of the bivariate survival function of the ACBVE model using a classical approach. Achcar and Santander (1993) performed Bayesian estimation of the ACBVE model using approximation methods constructed by Tierney and Kadane (1986). Achcar and Leandro (1998) used MCMC methods to estimate a bivariate exponential model. A good synthesis is given by Klein (1995) for this model. More recently, Hanagal and Ahmadi (2009) adopted Bayesian empirical approach of the bivariate exponential problem.

In this paper, we are interested by estimating the parameters and the MTBF of a bivariate exponential model. We use the classical maximum likelihood and the Bayesian approaches using noninformative and conjugate priors. The quadratic loss function is largely used in the literature.

Our aim is to prove, with an exhaustive Monte Carlo study, that it is possible to improve the maximum likelihood estimation using a Bayesian method and a suitable loss function. That's why, we propose to use a large variety of loss functions.

Four sections are proposed. The first section presents the genesis of the problem. In the second section, the Bayesian estimation of the parameters and the MTBF using noninformative prior is proposed. The third section proposes the use of the conjugate prior. Finally, a Monte Carlo study is performed in the last section where a comparative study of estimators is proposed using Pitman closeness and relative efficiency criterions. Then, we prove that, using a suitable loss function, we can obtain the best estimator via Bayesian method.

\section{Genesis of the model and the maximum likelihood estimation}

\subsection{Genesis of the model}

Many bivariate models are derived from the exponential distribution. The Freund (1961) distribution gives the joint distribution of the system with two components $\mathrm{A}$ and $\mathrm{B}$ with lifetimes $X$ and $Y$, when they run separately, are distributed according to $\operatorname{Ex}(\alpha)$ and $\operatorname{Ex}(\beta)$ respectively.

Moreover, in the case of simultaneous runs, when a failure occurs in A (resp. B), the lifetime of $\mathrm{B}$ (resp. A) is $\operatorname{Ex}\left(\alpha^{\prime}\right)\left(\right.$ resp.exp $\left.\left(\beta^{\prime}\right)\right)$ with $\alpha^{\prime}>\alpha$ and $\beta^{\prime}>\beta$.

The bivariate exponential distribution family of Block and Basu (1974) with parameters $\left(\lambda_{1}, \lambda_{2}, \lambda_{12}\right)$ is a particular parametrization of Freund corresponding to

$$
\begin{aligned}
\alpha & =\lambda_{1}+\lambda_{12}\left\{\lambda_{1} /\left(\lambda_{1}+\lambda_{2}\right)\right\} ; \alpha^{\prime}=\lambda_{1}+\lambda_{2} \\
\beta & =\lambda_{2}+\lambda_{12}\left\{\lambda_{2} /\left(\lambda_{1}+\lambda_{2}\right)\right\} ; \beta^{\prime}=\lambda_{2}+\lambda_{12} \\
\lambda_{1}, \lambda_{2}, \lambda_{12} & \geq 0
\end{aligned}
$$

The density $f(x, y)$ given by Block and Basu (1974) is

Journal home page: www.jafristat.net 


$$
f(x, y)=\left\{\begin{array}{l}
\frac{\lambda_{1} \lambda^{\prime}\left(\lambda_{2}+\lambda_{12}\right)}{\lambda_{1}+\lambda_{2}} \exp \left(-\lambda_{1} x-\left(\lambda_{2}+\lambda_{12}\right) y \text { if } x<y\right. \\
\frac{\lambda_{2} \lambda^{\prime}\left(\lambda_{2}+\lambda_{12}\right)}{\lambda_{1}+\lambda_{2}} \exp -\left(\left(\lambda_{1}+\lambda_{12}\right) x-\lambda_{2} y \text { if } x>y\right.
\end{array}\right.
$$

where $\lambda^{\prime}=\lambda_{1}+\lambda_{2}+\lambda_{12}$.

The model used in this work is strongly connected to the two above models assuming in the Freund model identical components, $\alpha=\beta=\lambda$ et $\alpha^{\prime}=\beta^{\prime}=\lambda \theta$. A reparametrization of (1.1) assuming $\lambda_{1}=\lambda_{2}=\lambda(2-\theta)$ and $\lambda_{12}=2 \lambda(\theta-1)$ leads to the density

$$
f(x, y)=\left\{\begin{array}{lll}
\theta \lambda^{2} \exp -2 \lambda x-\theta \lambda(y-x) & \text { si } & x<y \\
\theta \lambda^{2} \exp -2 \lambda y-\theta \lambda(x-y) & \text { si } & x>y
\end{array} \quad \lambda>0 ; \theta>0\right.
$$

Remark 1. 1. When $\theta \geq 1$, we obtain the Freund model.

2. If $1 \leq \theta \leq 2$ we obtain Block et Basu's model.

The function $f(x, y)$ given in $(2)$ is a density in $\mathbb{R}^{2}$. Assume that

$$
\begin{aligned}
U & =\min (X, Y) \\
V & =\max (X, Y) \\
W & =V-U
\end{aligned}
$$

Then, the density of $(U, V)$ is

$$
f_{U, V}(u, v)=2 \theta \lambda^{2} \exp (-2 \lambda u-\lambda \theta(v-u)) \quad \text { for } \quad 0<u<v
$$

and $(U, W)$ is distributed according to the density

$$
f_{U, W}(u, w)=2 \theta \lambda^{2} \exp (-2 \lambda u-\lambda \theta w) \quad \text { for } \quad u>0 ; w>0
$$

Hence, one can say that $U$ and $W$ are independent with $U \sim E x(2 \lambda)$ and $W \sim E x(\lambda \theta)$.

In our model, the lifetime corresponds to $V=\max (X, Y)$ obtained as follows

$$
f_{V}(v / \lambda, \theta)= \begin{cases}\frac{2 \theta \lambda}{(\theta-2)}\{\exp (-2 \lambda v)-\exp (-\lambda \theta v)\} & \text { if } \theta \neq 2 \\ 4 \lambda^{2} \int_{0}^{v} \exp \{-2 \lambda u-2 \lambda(v-u)\} d u=4 \lambda^{2} v \exp (-2 \lambda v) & \text { if } \theta=2\end{cases}
$$

Note that, when $\theta=2, V$ is distributed according to $\operatorname{Gamma}(2,2 \lambda)$ and the MTBF is

$$
T_{0}= \begin{cases}E(V / \lambda, \theta)=\int_{0}^{\infty} v f_{V}(v / \lambda, \theta) d v=\frac{(2+\theta)}{2 \lambda \theta} & \text { if } \theta \neq 2 \\ \frac{1}{\lambda} & \text { if } \theta=2\end{cases}
$$

In the reliability point of view, $E(V / \lambda, \theta)$ is the MTBF $T_{0}$. In the following, we consider the case $\theta \neq 2$ only.

In this work, we consider the estimation of the parameters and the MTBF denoted $T_{0}$ in the bivariate model given above. Using Monte carlo study, we perform the maximum likelihood approach and the Bayesian methodology and compare the estimators with respect to various loss functions. 
A. Chadli, H. Talhi and H. Fellag, Afrika Statistika, Vol. 8, 2013, pages 499-514. Comparison of the maximum likelihood and Bayes estimators for symmetric bivariate exponential distribution under different loss functions.

\subsection{Maximum likelihood estimation}

Consider the couples of observations $\left\{\left(u_{i}, w_{i}\right) ; i=1, \ldots n\right\}$ where $u_{i}=\min \left(x_{i}, y_{i}\right)$ and $w_{i}=$ $\left|x_{i}-y_{i}\right|$. The likelihood function is

$$
L(\underline{u}, \underline{w} / \lambda, \theta)=\prod_{i=1}^{n} f_{U, W}\left(u_{i}, w_{i}\right)=2^{n} \theta^{n} \lambda^{2 n} \exp \left(-2 \lambda S-\lambda \theta S^{\prime}\right),
$$

where $S=\sum_{i=1}^{n} u_{i}$ and $S^{\prime}=\sum_{i=1}^{n} w_{i}$.

Then, the MLE estimators $\lambda_{M V}$ and $\theta_{M V}$ of $\lambda$ and $\theta$ respectively are

$$
\lambda_{M V}=\frac{n}{2 S} \quad \text { et } \quad \theta_{M V}=\frac{2 S}{S^{\prime}}
$$

Note that $S$ and $S^{\prime}$ are independent and distributed according to $\operatorname{Gamma}(n, 2 \lambda)$ and $\operatorname{Gamma}(n, \lambda \theta)$.

Hence, one can deduce that $E\left(\lambda_{M V}\right)=\frac{n}{n-1} \lambda$ and $E\left(\theta_{M V}\right)=\frac{n}{n-1} \theta$.

We can add that these estimators are asymptotically unbiased. To obtain the MTBF, we have just to replace $\lambda$ and $\theta$ by $\lambda_{M V}$ and $\theta_{M V}$ in the expression (6) of $T_{0}$. We obtain

$$
T_{0, M V}=\frac{S+S^{\prime}}{n}
$$

\section{Bayesian estimation with vague prior}

In this section we propose to perform the estimation using Bayesian methods. Recall that a Bayesian estimator $d$ of a paramater $t$ minimizes the posterior cost with respect to a loss function $l(t ; d)$. The comparison of the MLE and the bayesian estimator is considered by many authors. For example, in Ahmed et al. (2010), the maximum likelihood estimation, Bayesian using Jeffrey prior and the extension of Jeffrey prior information for estimating the parameters of Weibull distribution of life time are presented. A simulation study comparison is made on the performance of these estimators. For more details on loss functions, one can see Congdon (2006). In this section, we consider the following loss functions.

\subsection{Loss functions}

\subsubsection{Quadratic loss function}

This loss function defined by $L_{1}(t, d)=(t-d)^{2}$ is proposed by Legendre (1805) and Gauss (1810) and it is the most used function in the literature. Then, the Bayesian estimator of $t$ is the posterior mean $\hat{d}_{B}=E(t / x)$.

\subsubsection{DeGroot loss function}

This function,, introduced by DeGroot (1970), is defined as follows

$$
L_{2}(\theta, d)=\left(\frac{t-d}{d}\right)^{2} \Longrightarrow \hat{d}_{B}=\frac{E\left(t^{2} / x\right)}{E(t / x)}
$$


A. Chadli, H. Talhi and H. Fellag, Afrika Statistika, Vol. 8, 2013, pages 499-514. Comparison of the maximum likelihood and Bayes estimators for symmetric bivariate exponential distribution under different loss functions.

\subsubsection{Linex loss function}

It's an asymetric function introduced by Varian (1975) and defined by

$$
L_{3}(t, d) \propto e^{a \Delta}-a \Delta-1, a \neq 0,
$$

with $a \neq 0$ and $\Delta=(d-t)$. Then, the Bayesian estimator is

$$
\hat{d}_{B}=-\frac{1}{a} \ln \left(E\left(e^{-a t}\right)\right)
$$

Note that when $a \rightarrow 0$, we obtain the quadratic loss function (see Zellner, 1986).

\subsubsection{Entropy loss function}

Calabria and Pulcini (1994) deduced a new loss function from the Linex as follows

$$
L_{4}(t, d)=\left(\frac{d}{t}\right)^{p}-p \ln \left(\frac{d}{t}\right)-1 \Longrightarrow \hat{d}_{B}=\left[E\left(\theta^{-p}\right)\right]^{-1 / p}
$$

In the Bayesian context, when we have few or no information of the parameter, we use vague priors. the most popular is due to Jeffreys (1961) defined as follows

$$
p(\lambda, \theta)=\left|I_{n}(\lambda, \theta)\right|^{1 / 2}=\frac{n}{\lambda \theta} .
$$

Notice that $\lambda$ and $\theta$ are independent.

\subsection{Parameter estimation}

The posterior density of $(\lambda, \theta)$ is

$$
p(\lambda, \theta / \underline{u}, \underset{-}{w})=\frac{(2 S)^{n}}{\Gamma(n)} \frac{\left(S^{\prime}\right)^{n}}{\Gamma(n)} \lambda^{2 n-1} \theta^{n-1} \exp (-2 S \lambda) \exp \left(-\lambda \theta S^{\prime}\right) .
$$

Then, one can say that the posterior densities of $\lambda$ and $\theta$ are $\operatorname{Gamma}(n, 2 S)$ and $\operatorname{Gamma}\left(n, \lambda S^{\prime}\right)$; respectively. Also, notice that $\frac{\theta S^{\prime}}{2 S}$ is distributed according to Fisher distribution $F(2 n, 2 n)$ and the conditional distribution $\lambda / \theta$ is $\operatorname{Gamma}\left(2 n, 2 S+\theta S^{\prime}\right)$.

Now, let us give the Bayesian estimators obtained with the various loss functions. Using the quadratic loss function, we obtain the bayesian estimators $\lambda_{v, Q}$ et $\theta_{v, Q}$ of $\lambda$ and $\theta$ respectively as follows

$$
\lambda_{v, Q}=\int_{0}^{\infty} \int_{0}^{\infty} \lambda p(\lambda, \theta \underset{-}{u}, \underset{-}{w}) d \lambda d \theta=\frac{n}{2 S}
$$

and

$$
\theta_{v, Q}=\int_{0}^{\infty} \int_{0}^{\infty} \theta p(\lambda, \theta / \underline{u}, \underset{-}{u}) d \lambda d \theta=\frac{n}{(n-1)} \frac{2 S}{S^{\prime}}
$$


A. Chadli, H. Talhi and H. Fellag, Afrika Statistika, Vol. 8, 2013, pages 499-514. Comparison of the maximum likelihood and Bayes estimators for symmetric bivariate exponential distribution under different loss functions.

Remark 2. These estimators are the same than those obtained with maximum likelihood approach. However, for $\theta$, the equality is true in the asymptotic case only. Indeed, $\lambda_{v}=\lambda_{M V}$ and $\theta_{v}=\frac{n}{n-1} \theta_{M V}$.

Under the DeGroot loss function, the Bayesian estimators $\lambda_{v, G}$ and $\theta_{v, Q}$ are equal to

$$
\begin{gathered}
\lambda_{v, G}=\frac{E\left(\lambda^{2} / \underline{u}, \underline{w}\right)}{E(\lambda / \underline{u}, \underline{w})}=\frac{(n+1)}{2 S}, \\
\theta_{v, G}=\frac{E\left(\theta^{2} / \underline{u}, \underline{w}\right)}{E(\theta / \underline{u}, \underline{w})}=\frac{(n+1)}{(n-2)} \frac{(2 S)}{S^{\prime}} .
\end{gathered}
$$

Under the Linex loss function defined by Varian (1975) the Bayesian estimator of $\lambda$ is

$$
\lambda_{v, L}=-\frac{1}{a} \log E(\exp (-a \lambda)) \quad \text { where } \quad E\left(e^{-a \lambda}\right)=\frac{\Gamma(n)}{S^{n}} \frac{\Gamma(n)}{(a+2 S)^{n}}
$$

Then,

$$
\lambda_{v, L}=-\frac{n}{a} \ln \frac{(2 S)}{(a+2 S)}
$$

Moreover, we obtain the Bayesian estimator $\theta_{v, L}$ of $\theta$ given by the equation

$$
\theta_{v, L}=-\frac{1}{a} \log E(\exp (-a \theta))
$$

After some computations, we obtain

$$
\theta_{v, L}=\frac{(2 S)^{n}\left(S^{\prime}\right)^{n}}{\Gamma(n)} \Psi_{1}(a, n) \quad \text { where } \quad \Psi_{1}(a, n)=\int_{0}^{\infty} \frac{\lambda^{2 n-1}}{\left(\lambda S^{\prime}+a\right)^{n}} \exp (-2 \lambda S) d \lambda
$$

Finally, under the entropy loss function, the Bayesian estimator of $\theta$ is

$$
\theta_{v, E}=\left[\frac{(2 S)^{n}}{\Gamma(n)} \frac{\left(S^{\prime}\right)^{n}}{\Gamma(n)} \frac{\Gamma(n-p)}{\left(S^{\prime}\right)^{n-p}} \frac{\Gamma(n+p)}{(2 S)^{n+p}}\right]^{-\frac{1}{p}}=\left[\frac{\Gamma(n) \Gamma(n)}{\Gamma(n+p) \Gamma(n-p)}\right]^{\frac{1}{p}} \frac{(2 S)}{S^{\prime}}
$$

Using the same method, we obtain the Bayesian estimator of $\lambda$

$$
\lambda_{v, E}=\frac{[(n-1)(n-2) \ldots(n-p)]^{1 / p}}{(2 S)}
$$


A. Chadli, H. Talhi and H. Fellag, Afrika Statistika, Vol. 8, 2013, pages 499-514. Comparison of the maximum likelihood and Bayes estimators for symmetric bivariate exponential distribution under different loss functions.

\subsection{Estimation of the $M T B F$}

Under the quadratic loss function, the Bayesian estimator of $T_{0}$ given in (1.6) is

$$
T_{0, v}=\frac{S^{\prime}}{(n-1)}+\frac{1}{2} \frac{2 S}{(n-1)}=\frac{S^{\prime}+S}{(n-1)}
$$

Note that (19) is close to the expression of $T_{0, M V}$.

Under the DeGroot loss function, we obtain the estimator $T_{0, v, G}$

$$
T_{0, v, G}=\frac{E\left(T_{0}^{2} / \underline{u}, \underline{w}\right)}{E\left(T_{0} / \underline{u}, \underline{w}\right)}=\frac{\left(S^{2}+S^{\prime 2}\right)}{(n-2)\left(S+S^{\prime}\right)}+\frac{S S^{\prime}}{(n-1)\left(S+S^{\prime}\right)}
$$

But, under Linex function, the estimator $T_{0, v, L}$ is given by the expression

$$
T_{0, v, L}=-\frac{1}{a} \ln E\left(\exp \left(-a T_{0}\right)\right)=-\frac{1}{a} \frac{(2 S)^{n}\left(S^{\prime}\right)^{n}}{\beta(n, n)} \Psi_{2}(a, n)
$$

where $\Psi_{2}(a, n)=\int_{0}^{\infty} \frac{\theta^{n-1}(2+\theta)^{2 n}}{\left[S^{\prime} \theta^{2}+2\left(S+S^{\prime}+a\right) \theta+4 S\right]^{2 n}} d \theta$ and $\beta(n, n)$ is the Beta function. Also, one can write

$$
\left(\frac{1}{T_{0}}\right)_{v, L}=-\frac{1}{a}\left[n \ln (2 S)+n \ln \left(S^{\prime}\right)-\ln \beta(n, n)+\ln \Psi_{2}(a, n)\right] .
$$

Finally, under the entropy loss function, the Bayesian estimator of $T_{0}$ is

$$
T_{0, v, E}=\left[E\left(\left(T_{0}\right)^{-p}\right)\right]^{-1 / p}=\frac{1}{2}\left[(2 S)^{n}\left(S^{\prime}\right)^{n} \frac{\Gamma(2 n+p)}{\Gamma(n) \Gamma(n)} \Psi_{3}(p, n)\right]^{-1 / p}
$$

where $\Psi_{3}(p, n)=\int_{0}^{\infty} \frac{\theta^{n+p-1}}{\left[(2+\theta)^{p}\left(2 S+\theta S^{\prime}\right)^{2 n+p}\right]} d \theta$

Since $\Psi_{1}, \Psi_{2}$ and $\Psi_{3}$ are untractable analytically, the computation of these integrals can be made using Monte Carlo methods.

\section{Bayesian estimation with conjugate prior}

There exists a family of conjugate priors for $(\lambda, \theta)$, (see, e.g., Weier, 1981) where the marginal prior of $\lambda$ is $\operatorname{Gamma}\left(g_{1}, h_{1}\right)$ and the conditional prior distribution of $\theta$ given $\lambda$ is $\operatorname{Gamma}\left(g_{2}, \lambda h_{2}\right)$. This means that $\lambda \theta$ is $\operatorname{Gamma}\left(g_{2}, h_{2}\right)$. Also,

$$
p(\lambda)=\frac{h_{1}^{g_{1}}}{\Gamma\left(g_{1}\right)} \lambda^{g_{1}-1} \exp \left(-h_{1} \lambda\right) \quad \text { and } \quad p(\theta / \lambda)=\frac{\left(h_{2}\right)^{g_{2}}}{\Gamma\left(g_{2}\right)} \lambda(\lambda \theta)^{g_{2}-1} \exp \left(-h_{2} \lambda \theta\right) .
$$

Then, we obtain the density of $(\lambda, \theta)$ as follows

$$
p(\lambda, \theta) \propto \lambda^{g_{1}+g_{2}-1} \theta^{g_{2}-1} \exp \left(-\lambda h_{1}\right) \exp \left(-\lambda h_{2} \theta\right) .
$$


A. Chadli, H. Talhi and H. Fellag, Afrika Statistika, Vol. 8, 2013, pages 499-514. Comparison of the maximum likelihood and Bayes estimators for symmetric bivariate exponential distribution under different loss functions.

Remark 3. If $g_{1}=g_{2}=h_{1}=h_{2}=0$, we obtain the noninformative prior case. The posterior density of $(\lambda, \theta)$ is given by the formula

$$
\begin{aligned}
p(\lambda, \theta / \underline{u}, \underline{w}) & =\frac{L(\underline{u}, \underline{w} / \lambda, \theta) p(\lambda, \theta)}{\int_{0}^{\infty} \int_{0}^{\infty} L(\underline{u}, \underline{w} / \lambda, \theta) p(\lambda, \theta) d \theta} \\
& =\frac{H_{1}^{G_{1}} H_{2}^{G_{2}}}{\Gamma\left(G_{1}\right) \Gamma\left(G_{2}\right)} \lambda^{G_{1}+G_{2}-1} \exp \left(-\lambda H_{1}\right) \theta^{G_{2}-1} \exp \left(-\lambda \theta H_{2}\right),
\end{aligned}
$$

where $G_{1}=n+g_{1} ; H_{1}=2 S+h_{1} ; G_{2}=n+g_{2}$ and $H_{2}=S^{\prime}+h_{2}$.

It is easy to notice that the posterior density of $\lambda$ is $\operatorname{Gamma}\left(G_{1}, H_{1}\right)$ and the posterior conditional density of $\theta$ given $\lambda$ is $\operatorname{Gamma}\left(G_{2}, \lambda H_{2}\right)$. Also, the posterior density of $\lambda$ given $\theta$ is $\operatorname{Gamma}\left(G_{1}+G_{2}, H_{1}+\theta H_{2}\right)$.

\subsection{Parameter estimation}

Under the quadratic loss function, the Bayesian estimators $\lambda_{B, Q}$ and $\theta_{B, Q}$ are as follows

$$
\lambda_{B, Q}=\frac{G_{1}}{H_{1}}=\frac{n+g_{1}}{2 S+h_{1}}
$$

and

$$
\theta_{B, Q}=\frac{G_{2}}{G_{1}-1}\left(\frac{2 S+h_{1}}{S^{\prime}+h_{2}}\right)
$$

Remark 4. One can notice that $\lambda$ is $\operatorname{Gamma}\left(g_{1}, h_{1}\right)$ and $\frac{g_{1} h_{2}}{g_{2} h_{1}} \theta$ is distributed according to Fisher distribution $F\left(2 g_{2}, 2 g_{1}\right)$. In order to determine the values of the parameters $h_{1}$, $g_{1}, h_{2}$ et $g_{2}$, one has to solve a sytem of four equations.

If we use the DeGroot loss function, the estimators obtained are

$$
\begin{gathered}
\lambda_{B, G}=\frac{E\left(\lambda^{2} / \underline{u}, \underline{w}\right)}{E(\lambda / \underline{u}, \underline{w})}=\frac{\left(G_{1}+1\right)}{H_{1}}=\frac{\left(n+1+g_{1}\right)}{\left(2 S+h_{1}\right)} \\
\theta_{B, G}=\frac{E\left(\theta^{2} / \underline{u}, \underline{w}\right)}{E(\theta / \underline{u}, \underline{w})}=\frac{H_{1}\left(G_{2}+1\right)}{H_{2}\left(G_{1}-2\right)}=\frac{\left(2 S+h_{1}\right)}{\left(S^{\prime}+h_{2}\right)} \frac{\left(n+1+g_{2}\right)}{\left(n-2+g_{1}\right)} .
\end{gathered}
$$

But, under Linex loss function, the estimators are given by

$$
\lambda_{B, L}=\frac{G_{1}}{a} \ln \left(1+\frac{a}{H_{1}}\right)
$$


A. Chadli, H. Talhi and H. Fellag, Afrika Statistika, Vol. 8, 2013, pages 499-514. Comparison of the maximum likelihood and Bayes estimators for symmetric bivariate exponential distribution under different loss functions.

and

$$
\theta_{B, L}=-\frac{1}{a}\left(G_{1} \ln H_{1}+G_{2} \ln H_{2}-\ln \Gamma\left(G_{1}\right)+\ln \Phi_{1}(a, n)\right)
$$

where $\Phi_{1}(a, n)=\int_{0}^{\infty} \frac{\lambda^{G_{1}+G_{2-1}}}{\left(\lambda H_{2}+a\right)^{G_{2}}} \exp \left(-\lambda H_{1}\right) d \lambda$.

Finally, under the entropy loss function, we obtain

$$
\lambda_{B, E}=\frac{\left[\left(G_{1}-1\right)\left(G_{1}-1\right) \ldots\left(G_{1}-p\right)\right]^{1 / p}}{H_{1}}
$$

and

$$
\theta_{B, E}=\left[E\left(\theta^{-p}\right)\right]^{-1 / p}=\frac{H_{1}}{H_{2}}\left[\frac{\left(G_{2}-1\right)\left(G_{2}-1\right) \ldots\left(G_{2}-p\right)}{\left(G_{1}+p-1\right)\left(G_{1}+p-2\right) \ldots\left(G_{1}\right)}\right]^{1 / p}
$$

\subsection{Estimation of the $M T B F$}

Under the quadratic function, the Bayesian estimator of $T_{0, B}$ is

$$
T_{0, B}=\frac{1}{2} \frac{H_{1}}{G_{1}-1}+\frac{H_{2}}{G_{2}-1}=\frac{1}{2}\left(\frac{2 S+h_{1}}{n+g_{1}-1}\right)+\frac{S^{\prime}+h_{2}}{n+g_{2}-1} .
$$

When we use the DeGroot loss function, we have, after computations,

$$
T_{0, B, G}=\frac{\left[\frac{H_{2}^{2}}{\left(G_{2}-1\right)\left(G_{2}-2\right)}+\frac{H_{1} H_{2}}{\left(G_{1}-1\right)\left(G_{2}-1\right)}+\frac{1}{4} \frac{H_{1}^{2}}{\left(G_{1}-1\right)\left(G_{1}-2\right)}\right]}{\left[\frac{1}{2} \frac{H_{1}}{\left(G_{1}-1\right)}+\frac{H_{2}}{\left(G_{2}-1\right)}\right]} .
$$

Under the Linex loss function, the estimator is

$$
\begin{aligned}
\frac{1}{T_{0, B, L}} & =-\frac{1}{a} \ln E\left(\exp \left(-a \frac{1}{T_{0}}\right)\right) \\
& =-\frac{1}{a} \ln \left[\frac{H_{1}^{G_{1}} H_{2}^{G_{2}}}{B\left(G_{1}, G_{2}\right)} \int_{0}^{\infty} \frac{\theta^{G_{2}-1}(2+\theta)^{G_{1}+G_{2}}}{\left[H_{2} \theta^{2}+\left(2 a+H_{1}+2 H_{2}\right) \theta+2 H_{1}\right]^{G_{1}+G_{2}}} d \theta\right] \\
& =-\frac{1}{a}\left[G_{1} \ln H_{1}+G_{2} \ln H_{2}-\ln B\left(G_{1}, G_{2}\right)+\ln \Phi_{2}(a, n)\right],
\end{aligned}
$$

where $\Phi_{2}(a, n)=\int_{0}^{\infty} \frac{\theta^{G_{2}-1}(2+\theta)^{G_{1}+G_{2}}}{\left[H_{2} \theta^{2}+\left(2 a+H_{1}+2 H_{2}\right) \theta+2 H_{1}\right]^{G_{1}+G_{2}}} d \theta$

Finally, under the entropy loss function, we obtain the following Bayesian estimator

$$
T_{0, B, E}=\frac{1}{2} H_{1}^{-G_{1} / p} H_{2}^{-G_{2} / p}\left[\frac{\Gamma\left(G_{1}+G_{2}+p\right)}{\Gamma\left(G_{1}\right) \Gamma\left(G_{2}\right)}\right]^{-1 / p}\left[\Phi_{3}(n, p)\right]^{-1 / p}
$$

where $\Phi_{3}(n, p)=\int_{0}^{\infty} \frac{\theta^{G_{2}-1}}{(2+\theta)^{p}\left(H_{1}+\theta H_{2}\right)^{G_{1}+G_{2}+p}} d \theta$. 
A. Chadli, H. Talhi and H. Fellag, Afrika Statistika, Vol. 8, 2013, pages 499-514. Comparison of the maximum likelihood and Bayes estimators for symmetric bivariate exponential distribution under different loss functions.

\section{Monte-Carlo study}

In this section we propose to study the performance of the Bayesian estimators of the reliability function and the parameters under some various loss functions with respect to the MLE. An exhaustive monte carlo comparative study is performed using the loss functions given in the previous sections. To complete the study, we add the absolute loss function.

In the following tables, we present the results of the monte carlo study. We simulate $n$ values $(\mathrm{n}=10,20,30,50,100,250)\left(\lambda_{i}, \theta_{i}\right)$ from the posterior distribution of $(\lambda, \theta) / u, w$. In this study, the true values are $\lambda=2.3996$ and $\theta=0.73317$. Also, in this study, we assume that $g_{1}=g_{2}=1$ and $h_{1}=h_{2}=2$. Since the bias and mean square error are not adequate to compare classical and Bayesian estimators, we propose to perform the comparison using the Pitman closeness criterion (see, e.g; Pitman, 1937 and Fuller, 1982) and the relative efficiency (Pandey et al., 2011). These criterions are largely used in the literature. The Pitman criterion is considered in many papers. It was used by Sugiura (1984) for estimating the normal covariance matrix, and was discussed by Peddada and Khattree (1986) and Rao et al. (1986). Kubokawa (1989) gives a family of estimators closer than the sample mean of a population in the sense of Pitman. Fountain (2000) constructed generalized class of closeness criteria for the pairwise comparison of estimators including Pitman's measure of closeness. Jozani (2012) showed that the Pitman's measure of closeness comparison of estimators under the balanced loss function can be reduced to the Pitman comparison under the usual absolute error loss.

The Pitman closeness criterion and the relative efficiency are defined as follows:

Definition 1. An estimator $\hat{\theta}_{1}$ of a parameter $\theta$ dominates in the sens of Pitman closeness criterion an other estimator $\hat{\theta}_{2}$, if, for all $\theta \in \Theta$

$$
P_{\theta}\left[\left|\hat{\theta}_{1}-\theta\right|<\left|\hat{\theta}_{2}-\theta\right|\right]>0.5
$$

Definition 2. The relative efficiency of an estimator $\hat{\theta_{1}}$ with respect to the estimator $\hat{\theta_{2}}$ under the loss function $l(t, \theta)$ is defined by

$$
E f f=\frac{\frac{1}{N} \sum_{i=1}^{N} l\left(\hat{\theta}_{1}(i), \theta\right)}{\frac{1}{N} \sum_{i=1}^{N} l\left(\hat{\theta_{2}}(i), \theta\right)}
$$

where $\left\{\hat{\theta}_{1}(i), i=1, \ldots, N\right\}$ and $\left\{\hat{\theta}_{2}(i), i=1, \ldots, N\right\}$ are samples from $\hat{\theta}_{1}$ and $\hat{\theta}_{2}$ respectively.

\subsection{Estimation with vague prior}

When the prior is vague, the results of the comparative study are given below. In what follows, the Bayesian estimator is compared with the MLE. The Gibbs sampler is used since

$$
\begin{gathered}
\lambda / \theta \sim \operatorname{Gamma}\left(2 n, 2 S+\theta S^{\prime}\right) \\
\theta / \lambda \sim \operatorname{Gamma}\left(n, \lambda S^{\prime} .\right)
\end{gathered}
$$


A. Chadli, H. Talhi and H. Fellag, Afrika Statistika, Vol. 8, 2013, pages 499-514. Comparison of the maximum likelihood and Bayes estimators for symmetric bivariate exponential distribution under different loss functions.

\subsubsection{Results for the parameters}

The Table 1 presents the values of the Pitman criterion when we compare the Bayesian estimators with the MLE. Under every loss function, the probabilities are calculated with the Pitman formula such that, when it is greater than 0.5 , one can say that the corresponding Bayesian estimator is better. In the Table 2, the relative efficiency of this comparison is given. The comparison with respect to relative efficiency is done such that, when it is less than one, the Bayesian estimator is better.

\begin{tabular}{|c|c|ccccc|}
\hline parameter & $n$ & squared loss & absolute loss & DeGroot loss & Linex loss & Entropy loss \\
\hline \multirow{5}{*}{$\lambda$} & 10 & 0.520 & 0.493 & 0.410 & 0.463 & 0.413 \\
& 20 & 0.477 & 0.480 & 0.480 & 0.420 & 0.410 \\
& 30 & 0.47 & 0.433 & 0.520 & 0.423 & 0.410 \\
& 50 & 0.477 & 0.483 & 0.477 & 0.460 & 0.450 \\
& 100 & 0.480 & 0.473 & 0.507 & 0.463 & 0.453 \\
& 250 & 0.457 & 0.490 & 0.473 & 0.487 & 0.483 \\
\hline \multirow{6}{*}{$\theta$} & 10 & 0.443 & 0.420 & 0.337 & 0.733 & 0.427 \\
& 20 & 0.447 & 0.507 & 0.370 & 0.657 & 0.480 \\
& 30 & 0.443 & 0.497 & 0.38 & 0.583 & 0.467 \\
& 50 & 0.467 & 0.433 & 0.417 & 0.540 & 0.477 \\
& 100 & 0.467 & 0.490 & 0.417 & 0.537 & 0.490 \\
& 250 & 0.470 & 0.497 & 0.460 & 0.477 & 0.533 \\
\hline
\end{tabular}

Table 1. Pitman comparison of the estimators of $\lambda$ and $\theta$.

\begin{tabular}{|c|c|ccccc|}
\hline parameter & $n$ & squared loss & absolute loss & DeGroot loss & Linex loss & Entropy loss \\
\hline \multirow{5}{*}{$\lambda$} & 10 & 1.010 & 0.973 & 0.892 & 0.261 & 0.825 \\
& 20 & 0.993 & 0.991 & 0.928 & 0.610 & 0.918 \\
& 30 & 0.993 & 1.004 & 0.947 & 0.740 & 0.958 \\
& 50 & 0.997 & 0.997 & 0.965 & 0.843 & 0.985 \\
& 100 & 0.996 & 1.000 & 1.001 & 0.889 & 0.958 \\
& 250 & 0.998 & 1.000 & 1.005 & 0.949 & 0.981 \\
\hline \multirow{6}{*}{$\theta$} & 10 & 1.408 & 1.012 & 0.672 & 0.738 & 0.79 \\
& 20 & 1.201 & 1.002 & 0.813 & 0.938 & 0.873 \\
& 30 & 1.159 & 1.007 & 0.949 & 0.979 & 0.900 \\
& 50 & 1.107 & 1.011 & 0.952 & 1.010 & 0.971 \\
& 100 & 1.035 & 1.001 & 0.936 & 0.994 & 0.988 \\
& 250 & 1.031 & 1.000 & 0.985 & 1.012 & 0.997 \\
\hline
\end{tabular}

Table 2. Relative Efficiency comparison of the estimators of $\lambda$ and $\theta$. 
A. Chadli, H. Talhi and H. Fellag, Afrika Statistika, Vol. 8, 2013, pages 499-514. Comparison of the maximum likelihood and Bayes estimators for symmetric bivariate exponential distribution under different loss functions.

We remark that the Bayesian estimation of $\lambda$ is not better than the MLE in the Pitman sens (see Table 1). However, in terms of relative efficiency (see Table 2), the Linex and the entropy loss functions improve the bayesian estimation and perform well.

Concerning the estimation of $\theta$, the Linex function gives us suitable and better results than the maximum likelihood approach with respect to Pitman and relative efficiency criterions (Tables 1 and 2). This can be easly seen when $n$ is not large.

\subsubsection{Results for the MTBF}

Concerning the estimation of the mean time between failure, we have obtained the results given in the Tables 3 and 4 as follows

\begin{tabular}{|c|ccccc|}
\hline$n$ & squared loss & absolute loss & DeGroot loss & Linex loss & Entropy loss \\
\hline 10 & 0.420 & 0.460 & 0.363 & 0.440 & 0.617 \\
20 & 0.473 & 0.490 & 0.440 & 0.490 & 0.587 \\
30 & 0.497 & 0.523 & 0.480 & 0.517 & 0.597 \\
50 & 0.470 & 0.477 & 0.447 & 0.477 & 0.520 \\
100 & 0.487 & 0.507 & 0.473 & 0.493 & 0.530 \\
250 & 0.510 & 0.500 & 0.500 & 0.510 & 0.493 \\
\hline
\end{tabular}

Table 3. Pitman comparison of the estimators of the MTBF.

\begin{tabular}{|c|ccccc|}
\hline$n$ & squared loss & absolute loss & DeGroot loss & Linex loss & Entropy loss \\
\hline 10 & 1.516 & 1.068 & 0.934 & 1.276 & 0.975 \\
20 & 1.180 & 1.031 & 0.866 & 1.112 & 0.990 \\
30 & 1.064 & 1.004 & 0.845 & 1.036 & 0.984 \\
50 & 1.089 & 1.015 & 0.973 & 1.062 & 0.998 \\
100 & 1.036 & 1.006 & 0.972 & 1.025 & 0.998 \\
250 & 1.005 & 1.002 & 0.974 & 1.002 & 0.995 \\
\hline
\end{tabular}

Table 4. Relative Efficiency comparison of the estimators of the MTBF.

For the estimation of the MTBF, the entropy function performs better using the two criterions. One can notice that, when $n$ is large, the results are equivalent.

Finally, one can conclude that, for a noninformative prior, the Linex and the entropy loss functions are better than quadratic and absolute loss functions. Moreover, if we compare the classical and the Bayesian approach, one can conclude that the Linex function for the parameters and the entropy function for the MTBF give better results. 
A. Chadli, H. Talhi and H. Fellag, Afrika Statistika, Vol. 8, 2013, pages 499-514. Comparison of the maximum likelihood and Bayes estimators for symmetric bivariate exponential distribution under different loss functions.

\subsection{Estimation with conjugate prior}

Now, we suppose that we use the conjugate prior instead of noninformative one. Here, the method used is also the Gibbs Sampler since

$$
\begin{gathered}
\lambda / \theta \sim \operatorname{Gamma}\left(G_{1}+G_{2}, H_{1}+\theta H_{2}\right) \\
\theta / \lambda \sim \operatorname{Gamma}\left(G_{2}, \lambda H_{2}\right)
\end{gathered}
$$

\subsubsection{Results for the parameters}

The comparison with respect to Pitman closeness criterion is given in the Table 5. The results are as follows:

\begin{tabular}{|c|c|ccccc|}
\hline Parameter & $n$ & squared loss & absolute loss & DeGroot loss & Linex loss & Entropy loss \\
\hline \multirow{5}{*}{$\lambda$} & 10 & 0.433 & 0.413 & 0.717 & 0.380 & 0.357 \\
& 20 & 0.463 & 0.437 & 0.703 & 0.413 & 0.383 \\
& 30 & 0.483 & 0.473 & 0.643 & 0.457 & 0.443 \\
& 50 & 0.483 & 0.477 & 0.593 & 0.460 & 0.447 \\
& 100 & 0.513 & 0.510 & 0.600 & 0.497 & 0.493 \\
& 250 & 0.497 & 0.497 & 0.557 & 0.477 & 0.467 \\
\hline \multirow{6}{*}{$\theta$} & 10 & 0.340 & 0.427 & 0.240 & 0.470 & 0.877 \\
& 20 & 0.400 & 0.443 & 0.323 & 0.463 & 0.823 \\
& 30 & 0.410 & 0.453 & 0.370 & 0.450 & 0.777 \\
& 50 & 0.447 & 0.480 & 0.410 & 0.477 & 0.707 \\
& 100 & 0.483 & 0.500 & 0.453 & 0.490 & 0.660 \\
& 250 & 0.433 & 0.467 & 0.407 & 0.450 & 0.543 \\
\hline
\end{tabular}

Table 5. Pitman comparison of the estimators of $\lambda$ and $\theta$.

Notice that the DeGroot loss function gives us the best results than the MLE of the parameter $\lambda$. However, it is not the case for $\theta$ where the entropy loss is more suitable. For the two cases, despite of the popularity of the the quadratic loss, one can say that the estimation can be improved with respect to the classical approach using more suitable loss function. The results are as follows

Here, we can remark that the Bayesian estimation of $\lambda$ is better than the MLE for all the loss functions considered. But, for the parameter $\theta$, the entropy loss is the best.

\subsubsection{Results for the MTBF}

In the following, we give the results of the comparative estimation of the MTBF using the different loss functions given above. The Tables 7 and 8 present the results for the Pitman closeness and relative efficiency criterions respectively.

In the case of estimation of the MTBF, the Bayesian estimator is the best if the entropy loss function is considered with respect to the Pitman and relative efficiency criterions. 
A. Chadli, H. Talhi and H. Fellag, Afrika Statistika, Vol. 8, 2013, pages 499-514. Comparison of the maximum likelihood and Bayes estimators for symmetric bivariate exponential distribution under different loss functions.

\begin{tabular}{|c|c|ccccc|}
\hline Parameter & $n$ & squared loss & absolute loss & DeGroot loss & Linex loss & Entropy loss \\
\hline \multirow{5}{*}{$\lambda$} & 10 & 0.320 & 0.695 & 0.624 & 0.086 & 0.586 \\
& 20 & 0.601 & 0.827 & 0.779 & 0.384 & 0.831 \\
& 30 & 0.702 & 0.863 & 0.859 & 0.500 & 0.826 \\
& 50 & 0.792 & 0.911 & 0.904 & 0.642 & 0.862 \\
& 100 & 0.861 & 0.943 & 0.940 & 0.759 & 0.867 \\
& 250 & 0.954 & 0.976 & 0.984 & 0.915 & 0.976 \\
\hline \multirow{6}{*}{$\theta$} & 10 & 1.207 & 0.986 & 0.506 & 0.475 & 0.518 \\
& 20 & 1.235 & 0.999 & 0.708 & 0.952 & 0.737 \\
& 30 & 1.182 & 1.011 & 0.741 & 1.023 & 0.831 \\
& 50 & 1.136 & 1.015 & 0.841 & 1.042 & 0.899 \\
& 100 & 1.068 & 0.995 & 0.890 & 1.028 & 0.953 \\
& 250 & 1.067 & 1.011 & 1.012 & 1.040 & 0.983 \\
\hline
\end{tabular}

Table 6. Relative Efficiency of the estimators of $\lambda$ and $\theta$.

\begin{tabular}{|c|ccccc|}
\hline$n$ & squared loss & absolute loss & DeGroot loss & Linex loss & Entropy loss \\
\hline 10 & 0.420 & 0.480 & 0.387 & 0.460 & 0.693 \\
20 & 0.400 & 0.423 & 0.377 & 0.413 & 0.633 \\
30 & 0.437 & 0.443 & 0.430 & 0.440 & 0.607 \\
50 & 0.500 & 0.523 & 0.473 & 0.513 & 0.613 \\
100 & 0.430 & 0.440 & 0.413 & 0.430 & 0.523 \\
250 & 0.467 & 0.477 & 0.450 & 0.477 & 0.533 \\
\hline
\end{tabular}

Table 7. Pitman comparison of the estimators of the MTBF.

\begin{tabular}{|c|ccccc|}
\hline$n$ & squared loss & absolute loss & DeGroot loss & Linex loss & Entropy loss \\
\hline 10 & 1.051 & 0.897 & 0.620 & 0.917 & 0.680 \\
20 & 1.067 & 0.970 & 0.774 & 0.994 & 0.823 \\
30 & 1.035 & 0.974 & 0.833 & 0.990 & 0.875 \\
50 & 0.983 & 0.969 & 0.841 & 0.967 & 0.912 \\
100 & 1.039 & 1.000 & 0.992 & 1.019 & 0.965 \\
250 & 1.014 & 1.001 & 0.990 & 1.008 & 0.986 \\
\hline
\end{tabular}

Table 8. Relative Efficiency comparison of the estimators of the MTBF.

\section{Conclusion}

This work shows that the maximum likelihood estimation of the parameters and the mean time between failure of the bivariate exponential distribution can be improved using Bayesian method. Also, we prove that this improvement can be efficient using a suitable loss function. For future prospects, one can construct a mixture of the loss functions used in this paper to obtain optimal estimation. 
A. Chadli, H. Talhi and H. Fellag, Afrika Statistika, Vol. 8, 2013, pages 499-514. Comparison of the maximum likelihood and Bayes estimators for symmetric bivariate exponential distribution under different loss functions.

\section{References}

Achcar, J.A. and Leandro, R.A., 1998. Use of Markov Chain Monte-Carlo methods in a Bayesian analysis od the Block and Basu bivariate exponential distribution, Annals of the Institute of Statistical Mathematics, 50. 403-416.

Achcar, J.A. and Santander L.A.M., 1993. Use of approximate Bayesian methods for the Block and Basu bivariate exponential distribution, Journal of the Italian Statistical Society, 3, 233-250

Ahmed, O.M, Al-Kutubi, H.S. and Ibrahim, N.A., 2010. Comparison of the Bayesian and Maximum Likelihood Estimation for Weibull Distribution. Journal of Mathematics and Statistics, 6 (2): 100-104

Block, H.W. and Basu,A.P., 1974. A continuous Bivariate exponential extension. J.Amer.Statist.Assoc., Vol 69, 1031-1037.

Calabria R. and Pulcini, G., 1994. An Engineering Approach to Bayes estimation for the Weibull distribution. Micron Electron Reliab. 34. 789-802.

Congdon, P., 2006. Bayesian Statistical Modelling Second edition. Wiley Eds

DeGroot, M.H., 1970. Optimal Statistical Decisions. New-York, Mc-Graw-Hill.

Fountain, R.L., 2000. A class of closeness criteria. Communication in statistics, theory and methods, 29(8). 1865-1883.

Freund, J.A., 1961. bivariate Extension of the exponential distribution. J.Amer.Statist.Assoc. Vol 56, 971-977.

Fuller, W.A., 1982. Closeness of estimators. Encyclopedia of statistical sciences, Vol 2, Wiley.

Gauss, C.F., 1810. Least Squares method for the Combinations of Observations. Translated by J. Bertrand (1955). Mallet-Bachelier, Paris.

Hanagal, D.D. and Ahmadi, K.A., 2009. Bayesian estimation of the parameters of bivariate exponential distribution. Communications in Statistics - Simulation and Computation, 38. 1391-1413.

Jeffreys, H., (1961).Theory of probability, Oxford: Clarendon Press

Jozani, M.J, 2012. A note on Pitman's measure of closeness with balanced loss function. Statistics, 1-6

Klein, J.P., 1995. Inference for Multivariate Exponential Distributions, in N. Bala-Krishman and A.P. Basu eds, The Exponential Distributions-Theory Methods and Applications Gordon and Breach, 333-350.

Klein, J.P. and Basu, A.P., 1985. On estimating reliability for bivariate exponential distributions, Sankhya, Series B, 47. 346-353.

Kubokawa, T., 1989. Closer estimators of a commen mean in the sense of Pitman. Ann. Inst. Statist. Math. Vol. 41, no. 3. 477-484.

Legendre, A., 1805. New Methods for the Determination of Orbits of Comets Courcier, Paris.

Pandey, B.N., Nidhi, D. and Pulastya B., 2011. Comparison between Bayesian and maximum likelihood estimation of scale parameter in Weibull distribution with known shape under Linex loss function. Journal of Scientific Research,Vol. 55, 163-172,2011

Peddada, S.D. and Khattree, R., 1986. On Pitman nearness and variance of estimators, Comm. Statist. A-Theory Methods, 15. 3005-3017.

Pitman, E.J.G., 1937. The closest estimates of statistical parameters, Proc. Cambridge Phil. Soc. 33. 212-222. 
A. Chadli, H. Talhi and H. Fellag, Afrika Statistika, Vol. 8, 2013, pages 499-514. Comparison of the maximum likelihood and Bayes estimators for symmetric bivariate exponential distribution under different loss functions.

Rao, C. R., Keating, J.P. and Mason, R.L., 1986. The Pitman nearness criterion and its determination, Comm. Statist. A- Theory Methods, 15, 3173-3191.

Sugiura, N., 1984. Asymptotically closer estimators for the normal covariance matrix, $J$. Japan Statist. Soc. 14. 145-155.

Tierney, L. and Kadane, J.B., 1986. Accurate aproximations for posterior moments and marginal densities, J. Amer. Statist. Assoc. 81. 82-86.

Varian, H.R., 1975. A Bayesian Approach to real estate assessment.Amsterdam, North Holland. 195-208.

Weier, D.R., 1981. Bayes estimation for bivariate survival models besed on the exponential distributions, Communications in Statistics-Theory and Methods, 10. 1415-1427.

Zellner.A. 1986. Bayesian estimation and prediction using asymmetric loss function. Jour. Amer. Statist. Assoc. 81. 446-451. 